\title{
Phagocytosis of optically-trapped particles: delivery of the pure phagocytic signal
}

\author{
Maurice B Hallett ${ }^{1}$ \\ ${ }^{I}$ Neutrophil Signalling Group, School of Medicine, Cardiff University, CF14 4XN, UK \\ Cell Research (2006) 16:852-854. doi:10.1038/sj.cr.7310105; published online 16 November 2006
}

\begin{abstract}
Phagocytosis is a fundamental cell biological process exhibited by a wide variety of cell types from single cell organisms, which rely on this for feeding, to phagocytes in higher animals, which rely on specialised immune cells for combating infecting micro-organisms. In the immune system, both macrophages and neutrophils play roles as phagocytes. Neutrophils are often called "professional phagocytes" because of their remarkable capacity for phagocytosis, being able to internalise microscopic particles (diam 0.5-3 $\mu \mathrm{m}$ ) of virtually any surface material. The efficiency and speed of phagocytosis is, however, increased by coating the surface of the particles with opsonins such as antibodies or the complement component $\mathrm{C} 3 \mathrm{bi}$ (acting on $\beta 2$ integrin receptors), and $\mathrm{C} 3 \mathrm{bi}$-accelerated phagocytosis by neutrophils is the first line of defence by the innate immune system in vivo, operating in advance of the slower production of antibodies. Understanding the mechanism of phagocytosis is, therefore, clearly an important goal.
\end{abstract}

However, although the phenomenon of phagocytosis has been observed for at least 150 years, it has proved difficult to study the mechanism of phagocytosis by neutrophils and other phagocytes. A major problem arises from the difficulty in simply observing the phagocytic event in isolation and in its entirety from start to end. In some previous studies, phagocytosis has been studied as part of the process of chemotaxis with the cell moving towards the particle before engaging it. In these approaches, it is difficult to disentangle the phenomena of phagocytosis from adhesion and chemotaxis and there is also the possible cross-talk between chemotactic and phagocytosis signalling pathways. These problems would be overcome by presenting the stimulus at a defined time and at a defined location on the neutrophil. Although some previous micromanipulation-based methodologies have been developed for this (Figure 1A), these could be criticised because they involve physical contact between the cell undergoing phagocytosis and a surface other than the phagocytic particle [1,2]. For example, the presentation of particles by micropipettes requires the neutrophils to be adherent (or loosely adherent) to glass or another surface [1]. Similarly, holding the neutrophil in a glass micropipette, although preventing sedimentation onto a surface, involves the cell contacting the glass of the micropipette and also to the suction pressure within the micropipette [2]. Both may constitute other signals to the cell. In an elegant report by Suzuki et al. in this issue of Cell Research [3], these problems are overcome by using laser optical traps to hold the particle and the cell. The laser trap exerts a holding force on the cell and the particle which can hold the cell in suspension. Phagocytosis can thus be induced without the cell contacting another physical surface. The cell and particle are simply brought together "in suspension" by movement of the laser traps. The process of phagocytosis can thus be observed in the absence of other contact signals to the cell (other than the holding force from the laser trap). In this way, the events of binding, phagocytic cup formation, engulfment and completion of phagocytosis can be clearly defined in the absence of adhesion, surface deformation or chemotaxis. This should prove valuable for a number of future studies, particularly in those involving signalling events within the phagocyte that can be visualised in real time (eg $\mathrm{Ca}^{2}+$ and PIP2 and PIP3 accumulation). The temporal relationship between changes in these signals during phagocytosis $[1,4,5]$ could then be studied in the absence of potentially confounding signals.

One of the issues surrounding the study of phagocytosis has been in defining the cellular morphology changes that lead to phagocytosis. Several types of phagocytosis have been reported and it is widely accepted [eg ref 6] that some opsonins (eg Fc $\gamma R$ engagement of antibody) lead to the formation of protruding pseudopodia whereas other opsonins 
A

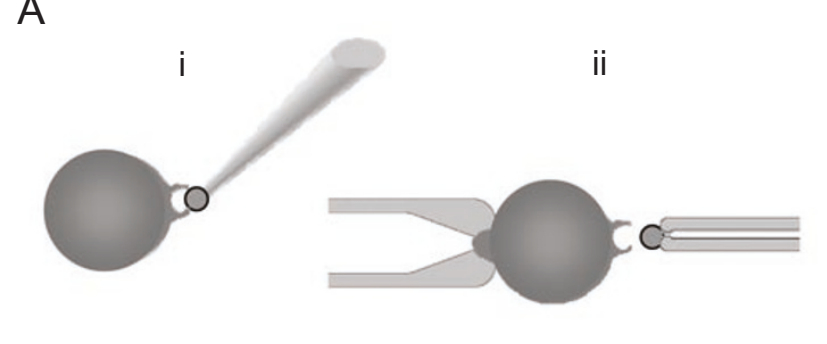

iii

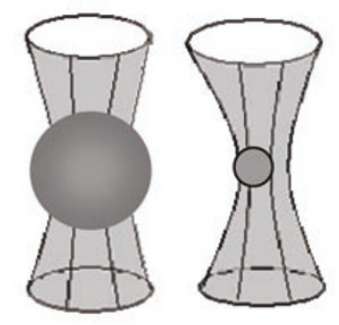

B

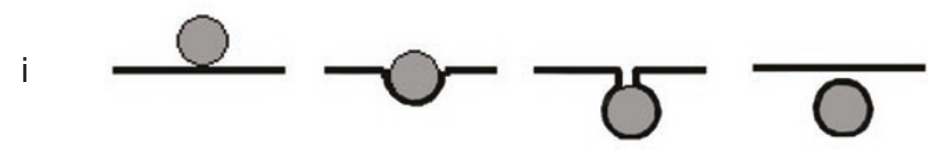

ii

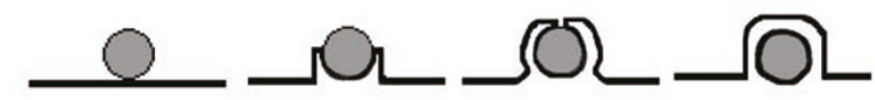

Figure 1 (A) Three methods used to present phagocytic stilumi to phagocytes (i) The pahagocytic stimulus is held by negative pressure applied through a narow bore micropipette and presented to a cell; while the phagocyte is spherical but attached to a surface. (ii) A large bore pipette is used to hold the phagocyte away from the surface and a second micropipette holding the phagocytic stimulus is used to present the stimulus to the cell. (iii) The two light paths shown represent the laser optical traps used to hold the phagocyte and the phagocytic stimulus. By moving the optical traps relative tro each other, the cell and particle are brought into contact. (B) The two types of phagocytosis are shown (i) "sinking phagocytosis" which does not involve protruding pseudopodia and (ii) conventional phagocytosis with extension of pseudopodia around the particle.

(eg $\beta 2$ integrin engagement) lead to a "sinking phagocytosis" (Figure 1B). These two phenomena are obviously very different and would require different molecular explanations. However, an important early finding from using the laser trap method is the demonstration that pseudopodia extension also occurs during phagocytosis mediated by $\beta 2$ integrin, despite it being traditionally thought of as a classic "sinking phagocytosis" stimulus. In fact, pseudopod extension by human neutrophils also occurs during $\beta 2$ integrin-mediated phagocytosis when the particle is delivered by micropipette $[1,5]$. It thus seems likely that the "sinking type" of phagocytosis is not related to the opsonin but may be dependent on the phagocyte-type being studied. In macrophages and cells with extensive endoplasmic reticulum (ER), a "sinking" type of phagocytosis may occur as the forming phagosome fuses with the internal membrane of sub-plasma membrane ER [7]. However, this has been disputed in macrophages [8]; and in neutrophils, the subject of this paper, there is no sub-plasma membrane ER at all. Neutrophils may thus be incapable of forming phagosomes without the extension of pseudopodia. In the laser trap method, reported in this issue, the neutrophil being held by the laser trap has little (or no) leverage, against which to push pseudopodia, apart from the particle attachment points. Thus "sinking phagocytosis", if it were possible, may be the energetically easier option. Thus, this early laser trap study [3] has already suggested that, in neutrophils at least, pseudopodia extension is the preferred route. It will be interesting to see whether this approach can be used with macrophages (or other phagocytic cells) and whether the "sinking" type of phagocytosis can be provoked by this method of stimulus presentation.

The development of laser trapping technology should allow the method reported here [3] to be extended and used in other labs. With this new way of presenting phagocytic stimuli to cells, it should now be possible to study signalling of the purely phagocytic event, uncontaminated by signals from unwanted stimuli to the cell. A new wave of advances in understanding the phagocytic mechanism and signalling is thus promised as laser traps become increasingly used to deliver the pure phagocytic signal. 


\section{References}

1 Dewitt $\mathrm{S}$, Hallett MB. Cytosolic free $\mathrm{Ca}^{2}+$ changes and calpain activation are required for beta2 integrin-accelerated phagocytosis by human neutrophils. J Cell Biol 2002; 159:181-189.

2 Herant M, Heinrich V, Dembo M. Mechanics of neutrophil phagocytosis: experiments and quantitative models . J Cell Sci 2006; 119: 1903-1913.

3 Suzuki T, Yanai M, Kubo H, Kanda A, Sasaki H, Butler JP. Interaction of non-adherent suspended neutrophils to complement opsonized pathogens: a new assay using optical traps. Cell Res 2006; 16: 887-894.

4 Yeung T, Terebiznik M, Yu LM, et al.Receptor activation alters inner surface potential during phagocytosis. Science 2006; 313:347351.

5 Dewitt $\mathrm{S}$, Tian W, Hallett MB. Localised PI $(3,4,5) \mathrm{P} 3$ at the phagocytic cup is required for both phagosome closure and $\mathrm{Ca}^{2}+$ signalling in HL60 neutrophils. J Cell Sci 2006; 119:443-451.

6 May RC, Machesky LM. Phagocytosis and the actin cytoskeleton. J Cell Sci 2001; 114:1061-1077.

7 Gagnon E, Duclos S, Rondeau C, et al. Endoplasmic reticulum-mediated phagocytosis is a mechanism of entry into macrophages Cell 2002; 110:119-131.

8 Touret N, Paroutis P, Terebiznik M, et al. Quantitative and dynamic assessment of the contribution of the ER to phagosome formation. Cell 2005; 123:157-170. 